\title{
Production and diagnostics of spin-polarized heavy ions in the sequential two-electron radiative recombination *
}

\author{
A. V. Maiorova ${ }^{\dagger}$, A. Surzhykov ${ }^{2,3,4}$, S. Tashenov ${ }^{2,3}$, V. M. Shabaev ${ }^{1}$, and T. Stöhlker ${ }^{3,4}$ \\ ${ }^{1} \mathrm{SPbSU}$, St. Petersburg, Russia; ${ }^{2} \mathrm{PI}$, Heidelberg, Germany; ${ }^{3} \mathrm{GSI}$, Darmstadt, Germany; ${ }^{4} \mathrm{HI}$, Jena, Germany
}

Within the last decades the radiative recombination (RR) by highly charged heavy ions remains the subject of intense theoretical and experimental research (see [1] and references therein). The RR is an effective tool for studying of the photoionization in the relativistic regime, which is not approachable directly at the present time. Moreover, the RR is interesting due to its sensitivity to the spin, relativistic and QED effects in the structure and dynamics of heavy atomic systems (see, e.g. [2, 3]).

In the recent years several experiments on researching the RR with polarized ion beams were proposed $[4,5]$. Furthermore, it was proposed to use the RR into polarized $\mathrm{H}$-like ions as the tool for beam spin diagnostic [6]. Information about the ion polarization is required for studying, for example, the parity nonconservation effects in highly charged ions or in heavy-ion collisions. But all of proposed experiments are hampered now by the lack of polarized ions.

In the present work we propose the method, that can be used for investigations of the RR into spin-polarized H-like ions. This approach is based on a subsequent capture of two electrons from two spatially separated targets by initial bare (finally He-like) ion and measurements of two emitted photons in coincidence. We choose the quantization axis (Z-axis) along the momentum $\mathbf{p}_{i}$ of the incoming electron. After the capture of the first electron, a photon is emitted in the direction $\mathbf{k}_{1}$, determined by polar angle $\theta_{1}$. It turns out that the relative magnetic sublevel population of the resulting $\mathrm{H}$-like ion depends on $\theta_{1}$. Hence the properties of the second photon, emitted in the direction $\mathbf{k}_{2}$ characterized by two angles $\left(\theta_{2}, \varphi_{2}\right)$, should be also dependent on $\theta_{1}$.

The population of the intermediate $\mathrm{H}$-like ion can be parameterized in terms of the polarization vector $\mathbf{P}=$ $\left(P_{x}, P_{y}, P_{z}\right)$. From the symmetry considerations in our case, when the photons, emitted in course of recombination of unpolarized electrons with bare ions, are observed in a particular setup, only single parameter $P_{y}$ is non-zero [7].

Information about the polarization of the H-like ions can be obtained from the analysis of the linear polarization of the second recombination photons. From the practical viewpoint it is more convenient to use the polarization ellipse parameters $P_{L}$ (the degree of linear polarization) and $\chi_{0}$ (the orientation of the principal axis with respect to the reaction plane) for the description of the x-ray linear polarization.

\footnotetext{
*Work supported by DFG, Helmholtz association, RFBR, FAIRRussia Research Center, Emmy Noether Programm and GSI.

† maiorova@pcqnt1.phys.spbu.ru
}

In Fig. 1, we display $P_{y}$ as a function of $\theta_{1}$ and $\chi_{0}$ as a function of $\theta_{2}$ in the case of $\varphi_{2}=90^{\circ}$. The calculations have been performed in the ion-rest frame for the incident electron energies $\varepsilon_{i}=109.7 \mathrm{keV}$, which correspond to the projectile energy $T_{p}=200 \mathrm{MeV} / \mathrm{u}$ in the laboratory frame. As one can see from the figure, the polarization of the $\mathrm{H}$ like ions following RR is very sensitive to the geometry of the photon emission. For example, a very significant degree of polarization, $P_{y} \sim 85 \%$, can be achieved for those ions, whose production is accompanied by the photon emission under the angle $\theta \simeq 150^{\circ}$. You can also see that $\chi_{0}$ shows strong dependence on the $\theta_{1}$ and, hence, on the degree of ion polarization. Thereby in the proposed scheme the characteristics of the first and the second RR photons are correlated through the spin states of intermediate H-like ions. Hence, using the proposed method we can "emulate" not only the production but also the diagnostics of heavy ion beams.
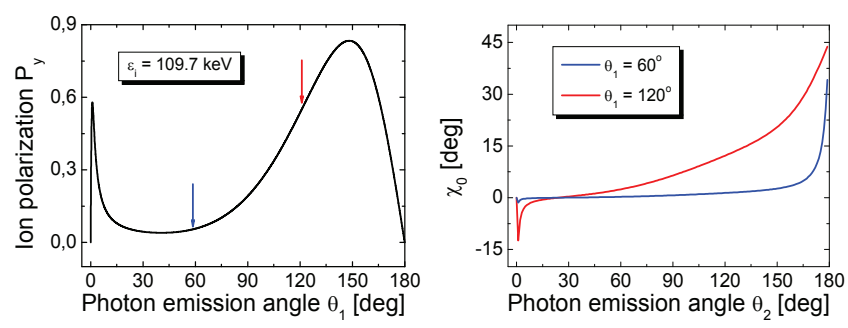

Figure 1: Component $P_{y}$ of the polarization vector of $\mathrm{H}$ like uranium ions $(Z=92)$ as a function of first photon emission direction $\theta_{1}$ (left panel) and angle $\chi_{0}$ as a function of the emission angle of the second photon $\theta_{2}$ at $\varphi_{2}=90^{\circ}$ (right panel). The calculations are performed in the ionrest frame for the kinetic energy $\varepsilon_{i}=109.7 \mathrm{keV}$ of the incoming electron.

\section{References}

[1] J. Eichler, Th. Stöhlkler, Phys. Rep. 439, 1 (2007).

[2] Th. Stöhlkler et al., Phys. Rev. Lett. 82, 3232 (1999).

[3] S. Tashenov et al., Phys. Rev. Lett. 97, 223202 (2006).

[4] A. Surzhykov et al. Phys. Rev. A 68, 022710 (2003).

[5] A. E. Klasnikov et al., NIMB 235, 284 (2005).

[6] A. Surzhykov et al. Phys. Rev. Lett. 94, 203202 (2005).

[7] V. V. Balashov et al., Polarization and Correlation Phenomena in Atomic Collisions (New York: Kluwer Academic, 2000). 